\title{
Structure and Properties of $\mathrm{Gd}_{3} \mathrm{Ge}_{4}$. The Orthorhombic
}

\section{$\mathrm{RE}_{3} \mathrm{Ge}_{4}$ Structures Revisited $(\mathrm{RE}=\mathrm{Y}, \mathrm{Tb}-\mathrm{Tm})$}

\author{
Paul H. Tobash, Gary DiFilippo, and Svilen Bobev* \\ Department of Chemistry and Biochemistry, University of Delaware, Newark, Delaware 19716
}

Namjung Hur, Joe D. Thompson, and John L. Sarrao

Materials Physics and Applications Division (MPA), Los Alamos National Laboratory, Los Alamos, New Mexico 87545

\section{Content}

Synthesis of $\mathrm{RE}_{3} \mathrm{Ge}_{4}(\mathrm{RE}=\mathrm{Y}, \mathrm{Gd}-\mathrm{Tm})$

Figure S1. Graphical representations of the experimental and the calculated powder X-ray diffraction patterns for $\mathrm{Gd}_{3} \mathrm{Ge}_{4}$. Since the crystals were grown in lead flux, elemental $\mathrm{Pb}$ is also present.

Figure S2. Powder diffraction patterns of arc-melted samples with nominal composition $\mathrm{Gd}_{3} \mathrm{Ge}_{4}$, annealed for 2 weeks at six different temperatures.

Figure S3. Contour diagram of the difference Fourier map and plots of a fragment of the structure of $\mathrm{Gd}_{3} \mathrm{Ge}_{4}$ drawn with anisotropic displacement parameters (90\% probability).

Figure S4. Coordination polyhedra around the $\mathrm{Gd}$ atoms in the structure of $\mathrm{Gd}_{3} \mathrm{Ge}_{4}$. The corresponding bond distances are listed in Table 3.

Figure S5. Temperature dependence of the magnetic susceptibility of polycrystalline $\mathrm{RE}_{3} \mathrm{Ge}_{4}(\mathrm{RE}=\mathrm{Y}$, Ho, Er, Tm) measured at an applied field of 500 Oe. The insets show the inverse susceptibility as a function of the temperature.

Table S1. The $h k l$ indices, their $2 \theta$ values and relative intensities for $\mathrm{Gd}_{3} \mathrm{Ge}_{4}$. 


\section{Supporting Information}

Table S2. Selected crystal data and structure refinement parameters for $R_{3} G_{4}$ compounds $(R E=Y$, Gd-Tm) taken at 120(2) K.

Table S3. Selected crystal data and structure refinement parameters for $R_{3} G_{4}$ compounds $(R E=Y$, Gd-Tm) taken at 293(2) K.

Table S4. Selected crystal data and structure refinement parameters for $\mathrm{Gd}_{3} \mathrm{Ge}_{4}$ in $C 2 / c$.

Table S5. Atomic coordinates and equivalent isotropic displacement parameters for $\mathrm{Y}_{3} \mathrm{Ge}_{4}$ at $120 \mathrm{~K}$.

Table S6. Atomic coordinates and equivalent isotropic displacement parameters for $\mathrm{Tb}_{3} \mathrm{Ge}_{4}$ at $120 \mathrm{~K}$.

Table S7. Atomic coordinates and equivalent isotropic displacement parameters for $\mathrm{Dy}_{3} \mathrm{Ge}_{4}$ at $120 \mathrm{~K}$.

Table S8. Atomic coordinates and equivalent isotropic displacement parameters for $\mathrm{Ho}_{3} \mathrm{Ge}_{4}$ at $120 \mathrm{~K}$.

Table S9. Atomic coordinates and equivalent isotropic displacement parameters for $\mathrm{Er}_{3} \mathrm{Ge}_{4}$ at $120 \mathrm{~K}$.

Table S10. Atomic coordinates and equivalent isotropic displacement parameters for $\mathrm{Tm}_{3} \mathrm{Ge}_{4}$ at $120 \mathrm{~K}$.

Table S11. Atomic coordinates and equivalent isotropic displacement parameters for $\mathrm{Y}_{3} \mathrm{Ge}_{4}$ at $293 \mathrm{~K}$.

Table S12. Atomic coordinates and equivalent isotropic displacement parameters for $\mathrm{Gd}_{3} \mathrm{Ge}_{4}$ at $293 \mathrm{~K}$.

Table S13. Atomic coordinates and equivalent isotropic displacement parameters for $\mathrm{Tb}_{3} \mathrm{Ge}_{4}$ at $293 \mathrm{~K}$.

Table S14. Atomic coordinates and equivalent isotropic displacement parameters for $\mathrm{Dy}_{3} \mathrm{Ge}_{4}$ at $293 \mathrm{~K}$.

Table S15. Atomic coordinates and equivalent isotropic displacement parameters for $\mathrm{Ho}_{3} \mathrm{Ge}_{4}$ at $293 \mathrm{~K}$.

Table S16. Atomic coordinates and equivalent isotropic displacement parameters for $\mathrm{Er}_{3} \mathrm{Ge}_{4}$ at $293 \mathrm{~K}$.

Table S17. Atomic coordinates and equivalent isotropic displacement parameters for $\mathrm{Tm}_{3} \mathrm{Ge}_{4}$ at $293 \mathrm{~K}$.

Table S18. Selected bond distances in $\mathrm{Y}_{3} \mathrm{Ge}_{4}$ (revised) at $120 \mathrm{~K}$.

Table S19. Selected bond distances in $\mathrm{Tb}_{3} \mathrm{Ge}_{4}$ (revised) at $120 \mathrm{~K}$.

Table S20. Selected bond distances in $\mathrm{Dy}_{3} \mathrm{Ge}_{4}$ (revised) at $120 \mathrm{~K}$.

Table S21. Selected bond distances in $\mathrm{Ho}_{3} \mathrm{Ge}_{4}$ (revised) at $120 \mathrm{~K}$.

Table S22. Selected bond distances in $\mathrm{Er}_{3} \mathrm{Ge}_{4}$ (revised) at $120 \mathrm{~K}$.

Table S23. Selected bond distances in $\mathrm{Tm}_{3} \mathrm{Ge}_{4}$ (revised) at $120 \mathrm{~K}$. 


\section{Supporting Information}

\section{Synthesis of $\mathrm{RE}_{3} \mathrm{Ge}_{4}(\mathrm{RE}=\mathrm{Y}, \mathrm{Tb}-\mathrm{Tm})$}

The flux reactions were carried out in $2 \mathrm{~cm}^{3}$ alumina crucibles, which were subsequently enclosed in fused silica ampoules and flamed-sealed under vacuum. The arc-melting was done with the aid of a custom-built furnace under argon and over a water-cooled copper hearth.

$G_{3} G e_{4} . \mathrm{Pb}$ flux: In this experiment, a reaction mixture containing the starting materials in a molar ratio $\mathrm{Gd}: \mathrm{Ge}: \mathrm{Pb}=1: 1: 5$ was heated at a rate of $300^{\circ} / \mathrm{h}$ to $1373 \mathrm{~K}$, allowed to equilibrate at this temperature for 1.5 hours and then cooled to $773 \mathrm{~K}$ at a rate of $30^{\circ} / \mathrm{h}$. At this point the reaction was taken out from the furnace and the molten lead metal (m.p. $601 \mathrm{~K}$ ) was removed by centrifugation. Further and more elaborate details on flux-growth procedure can be found elsewhere (Kanatzidis, M. G.; Pöttgen, R.; Jeitschko, W. Angew. Chem. Int. Ed. 2005, 44, 6996). The main products of this reaction turned out to be two phases crystallizing with different morphologies: irregular pieces of $\mathrm{Gd}_{3} \mathrm{Ge}_{4}$ and small cube-shaped crystals of $\mathrm{GdPb}_{3}$. The yield based on $\mathrm{Gd}_{3} \mathrm{Ge}_{4}$ was low (ca. 30-40\%) and in an attempt to improve it, different temperature profiles and metal fluxes were tried. These efforts proved unsuccessful - for example, the use of In metal as a flux produces exclusively the ternary compound $\mathrm{Gd}_{2} \mathrm{InGe}_{2}$ (Tobash, $\mathrm{P}$. H.; Lins, D.; Bobev, S.; Lima, A.; Hundley, M. F.; Thompson, J. D.; Sarrao, J. L. Chem. Mater. 2005, 17, 5567); Sn flux on the other hand affords a different ternary phase $\mathrm{GdSn}_{1-x} \mathrm{Ge}_{1+x}(0<x<0.2)$ with a narrow homogeneity range. The results of the latter studies will be discussed in a forthcoming publication. It should be noted that in no case were ternary phases of $\mathrm{Gd}-\mathrm{Pb}-\mathrm{Ge}$ ever discovered in the reactions. This is most certainly due to the fact of the larger size of $\mathrm{Pb}$ compared to In or $\mathrm{Sn}$.

In subsequent reactions aimed at synthesizing $\mathrm{Gd}_{3} \mathrm{Ge}_{4}$ in larger yield and without the use of metal flux,

the elements were loaded with the stoichiometric Gd : Ge ratio and arc-melted. Due to the high melting point of $\mathrm{Gd}(1586 \mathrm{~K})$, this technique was preferred to the conventional heating in welded niobium tubes. It was repeated 3 times and the weight-loss was below $c a .0 .5 \%$. The raw product of such reaction was a polycrystalline mixture of $\mathrm{GdGe}\left(\mathrm{CrB}\right.$-type) and poorly characterized $\mathrm{GdGe}_{2-x}\left(\mathrm{AlB}_{2}\right.$-derivatives, $0.25<x$ 


\section{Supporting Information}

< 0.4) (Tobash, P. H.; Lins, D.; Bobev, S.; Hur, N.; Thompson, J. D.; Sarrao, J. L. Inorg. Chem. 2006, 45,

7286). Afterwards, the "as-cast" powders were annealed for 2 weeks at temperatures between $773 \mathrm{~K}$ and $1273 \mathrm{~K}$. Such heat treatments, particularly those at $1073 \mathrm{~K}$ and $1173 \mathrm{~K}$ led to the formation of $\mathrm{Gd}_{3} \mathrm{Ge}_{4}$, in accordance with the results from earlier work on polycrystalline $\mathrm{RE}_{3} \mathrm{Ge}_{4}(\mathrm{RE}=\mathrm{Tb}-\mathrm{Tm})$. However, it should be explicitly noted that while $\mathrm{Gd}_{3} \mathrm{Ge}_{4}$ was formed under these conditions, it was never obtained as a pure phase - the product always contained residual $\mathrm{GdGe}$ and $\mathrm{GdGe}_{2-x}$.

$T b_{3} G e_{4}$. Indium flux was found inappropriate for the synthesis of $\mathrm{Tb}_{3} \mathrm{Ge}_{4}$ due to the formation of $\mathrm{Tb}_{2} \mathrm{InGe}_{2}$ of as a by-product. Therefore, to circumvent this problem, lead flux was used instead. The reactions were loaded with the corresponding elements in a ratio of $\mathrm{Tb}: \mathrm{Ge}: \mathrm{Pb}(1: 1: 5)$ and were rapidly heated to $1373 \mathrm{~K}$ (rate $300^{\circ} / \mathrm{h}$ ), allowed to equilibrate there for 1.5 hours, and then cooled to $773 \mathrm{~K}$ (rate $30^{\circ} / \mathrm{h}$ ) where the flux was quickly removed as outlined above. The products of such reaction were crystals with a needle-like morphology and silver-metallic luster $\left(\mathrm{Tb}_{3} \mathrm{Ge}_{4}\right)$, and a small amount of cubelike crystals of $\mathrm{TbPb}_{3}$. Because of this side-reaction and depleting the amount of rare-earth metal, it should be explicitly noted that the use of stoichiometric ratio of $\mathrm{Tb}:$ Ge here resulted in the synthesis of another, Ge-richer phase $\mathrm{Tb}_{3} \mathrm{Ge}_{5}$, analogous to $\alpha-\mathrm{Sm}_{3} \mathrm{Ge}_{5}$ (Tobash, P. H.; Lins, D.; Bobev, S.; Hur, N.; Hundley, M. F.; Thompson, J. D.; Sarrao, J. L. Inorg. Chem. 2006, 45, 7286). We also note that $\mathrm{Tb}_{3} \mathrm{Ge}_{4}$ can be synthesized by arc-melting the corresponding elements in the 3:4 ratio and annealing the mixture at $1073 \mathrm{~K}$ for 14-21 days as described in details elsewhere (Oleksyn, O. Ya.; Bodak, O. I. J. Alloys Compd. 1994, 210, 19; Janssen, Y.; Bruck, E.; de Groot, C. H.; Kayzel, F. E.; de Boer, F. R.; Buschow, K. H. J.; Oleksyn, O.; Schobinger-Papamantellos, P. J. Magn. Magn. Mater. 1998, 177, 1147).

$\mathrm{Dy}_{3} \mathrm{Ge}_{4}, \mathrm{Ho}_{3} \mathrm{Ge}_{4}$, and $\mathrm{Er}_{3} \mathrm{Ge}_{4}$. Reactions were loaded with the corresponding elements in a ratio of RE:Ge:In (1:1:10; RE = Dy, Ho, Er) and were rapidly heated to $1233 \mathrm{~K}$ (rate $300^{\circ} / \mathrm{h}$ ), allowed to equilibrate there for 1.5 hours and then slowly cooled to $773 \mathrm{~K}$ (rate $50^{\circ} / \mathrm{h}$ ). At this temperature which the reaction were quickly removed and the In was separated from the reaction product through 


\section{Supporting Information}

centrifugation. Note that this profile includes a faster cooling step, which proved to be critical in order to avoid the formation of $\mathrm{RE}_{2} \mathrm{InGe}_{2}$ (Tobash, P. H.; Lins, D.; Bobev, S.; Lima, A.; Hundley, M. F.; Thompson, J. D.; Sarrao, J. L. Chem. Mater. 2005, 17, 5567). In these three cases, small amounts of $\mathrm{REIn}_{3}$ were also formed but these phases were easily distinguishable by their cubic morphology. Lead flux reactions carried out under the same conditions produced much smaller crystals and in lower yields. As before, $\mathrm{Dy}_{3} \mathrm{Ge}_{4}, \mathrm{Ho}_{3} \mathrm{Ge}_{4}$ and $\mathrm{Er}_{3} \mathrm{Ge}_{4}$ crystallized with a needle-like morphology with silver-metallic luster. We also note that the latter can be synthesized by arc-melting the corresponding elements in the 3:4 ratio and annealing the mixture at $1073 \mathrm{~K}$ for $14-21$ days as described in details elsewhere (Oleksyn, O. Ya.; Bodak, O. I. J. Alloys Compd. 1994, 210, 19; Janssen, Y.; Bruck, E.; de Groot, C. H.; Kayzel, F. E.; de Boer, F. R.; Buschow, K. H. J.; Oleksyn, O.; Schobinger-Papamantellos, P. J. Magn. Magn. Mater. 1998, 177, 1147).

$\mathrm{Tm}_{3} G e_{4}$. Indium flux worked very well synthesis of $\mathrm{Tm}_{3} \mathrm{Ge}_{4}$. The flux reactions were loaded with the corresponding elements in a stoichiometric ratio of Tm:Ge:In (1:1:10) and were rapidly heated to $1233 \mathrm{~K}$ (rate $300^{\circ} / \mathrm{h}$ ), allowed to equilibrate there for 1.5 hours and then slowly cooled to $773 \mathrm{~K}$ (rate $30^{\circ} / \mathrm{h}$ ) in which the reaction vessel was quickly removed and the In was separated from the reaction product through centrifugation. The main product of such reactions was $\mathrm{Tm}_{3} \mathrm{Ge}_{4}$, which crystallizes as nicely faceted needle-like crystals with silver-metallic luster. In addition to the title compound, the reactions yielded crystals with plate-like morphologies, which were later identified as $\mathrm{TmGe}$ and $\mathrm{TmGe}_{2}$ ( $\mathrm{CrB}$ and $\mathrm{ZrSi}_{2}$-types, respectively). Fortunately, due to the very different morphologies of the title compounds and the side products $\left(\mathrm{Tm}_{3} \mathrm{Ge}_{4}\right.$-needles and $\mathrm{TmGe}, \mathrm{TmGe}_{2}$-plates), they were easily distinguishable from one another. Single crystals of $\mathrm{Tm}_{3} \mathrm{Ge}_{4}$ appear air and moisture stable.

$Y_{3} G e_{4}$. The corresponding elements in a stoichiometric ratio with a 10 -fold excess of In as a flux were loaded in alumina crucibles, enclosed in evacuated fused silica tubes. The reaction mixtures were heated to $1233 \mathrm{~K}$ (rate $300^{\circ} / \mathrm{h}$ ), allowed to equilibrate there for 1.5 hours and then slowly cooled to $773 \mathrm{~K}$ 


\section{Supporting Information}

(rate $30 \%$ h) at which time the reaction vessel was quickly removed and the In was separated from the reaction product through centrifugation. The crystals obtained from this reaction had a needle-like morphology and were proven by X-ray diffraction to be pure phase $\mathrm{Y}_{3} \mathrm{Ge}_{4}$.
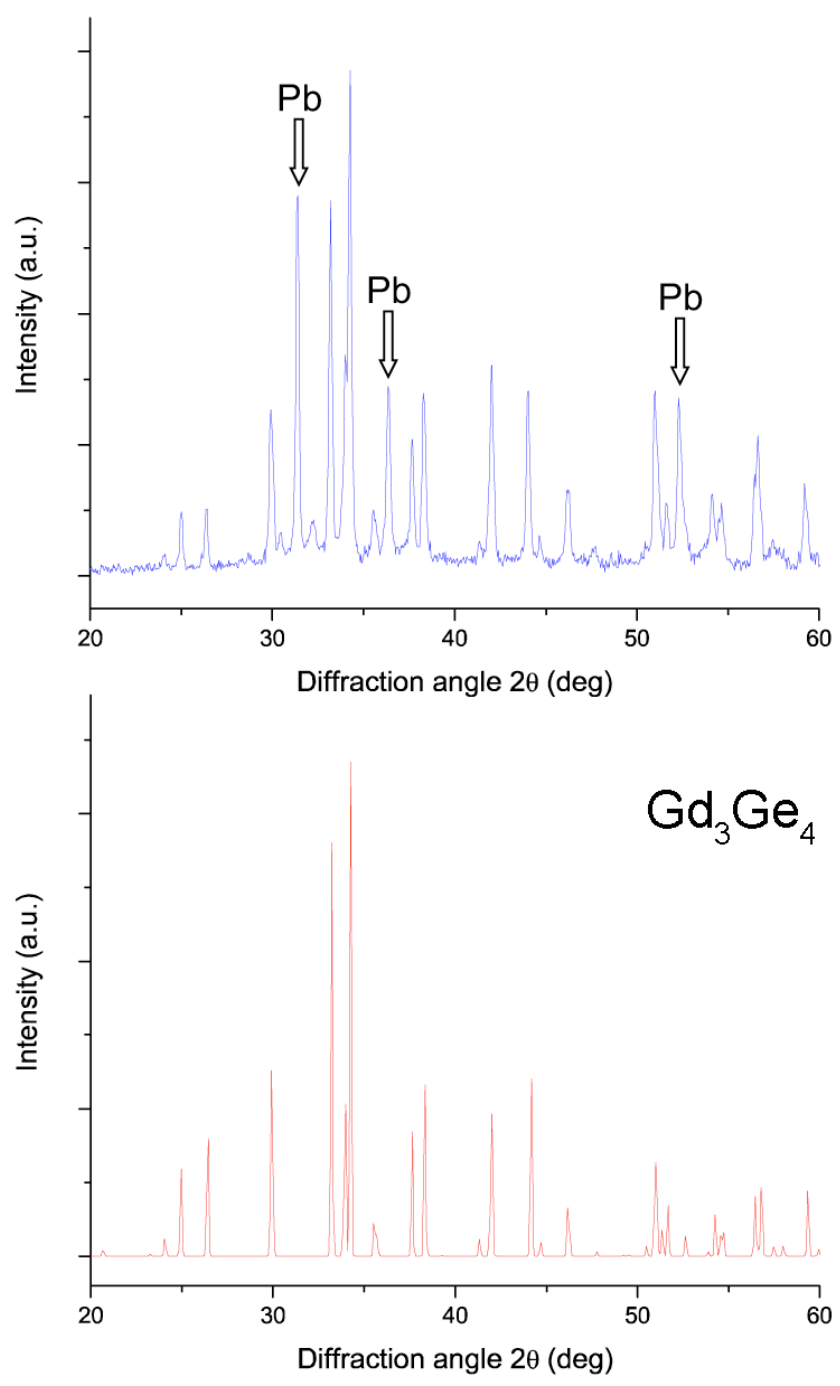

Figure S1

Graphical representations of the experimental and the calculated powder X-ray diffraction patterns for $\mathrm{Gd}_{3} \mathrm{Ge}_{4}$. Since the crystals were grown in lead flux, elemental $\mathrm{Pb}$ is also present. 


\section{Supporting Information}

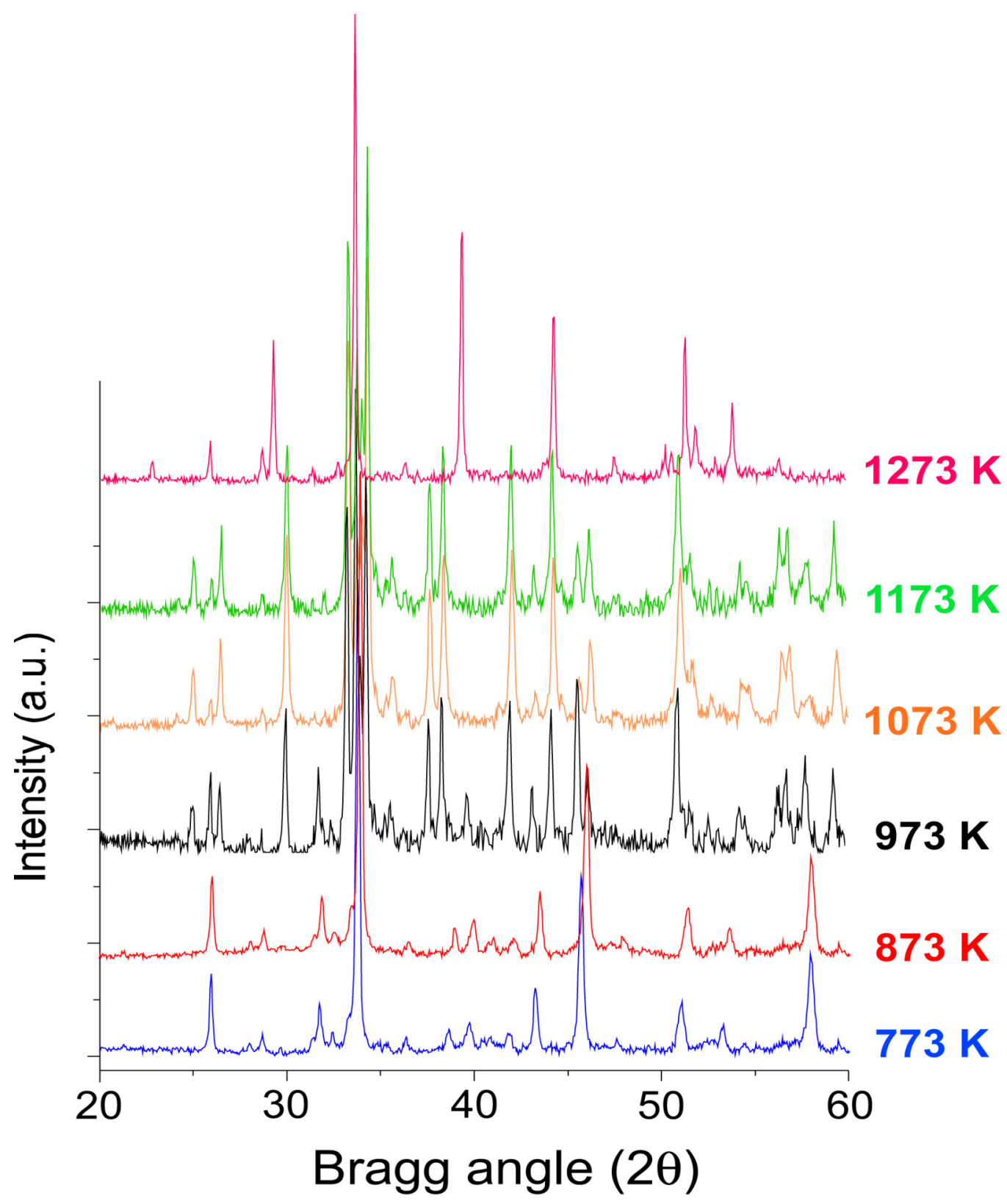

Figure S2

$\mathrm{Gd}_{3} \mathrm{Ge}_{4}$ synthesis was attempted through "on-stoichiometry" reactions using direct arc-melting. This led to a mixture of $\mathrm{GdGe}$ (minor) and $\mathrm{GdGe}_{2-x}$ (major) phases. Annealing at six temperatures for 2 week periods provided an indication that $\mathrm{Gd}_{3} \mathrm{Ge}_{4}$ may form through a peritectic reaction of $\mathrm{GdGe}$ and $\mathrm{GdGe}_{2-\mathrm{x}}$ between $1073 \mathrm{~K}$ and $1173 \mathrm{~K}$. As seen above, annealing at these conditions led to the formation of $\mathrm{Gd}_{3} \mathrm{Ge}_{4}$ (a small amount of $\mathrm{GdGe}_{2-x}$ is left). The samples annealed at temperatures below $1073 \mathrm{~K}$ revealed that such transformation did not take place. Above $1173 \mathrm{~K}$ the stable phase is $\mathrm{Gd}_{3} \mathrm{Ge}_{5}$. 


\section{Supporting Information}

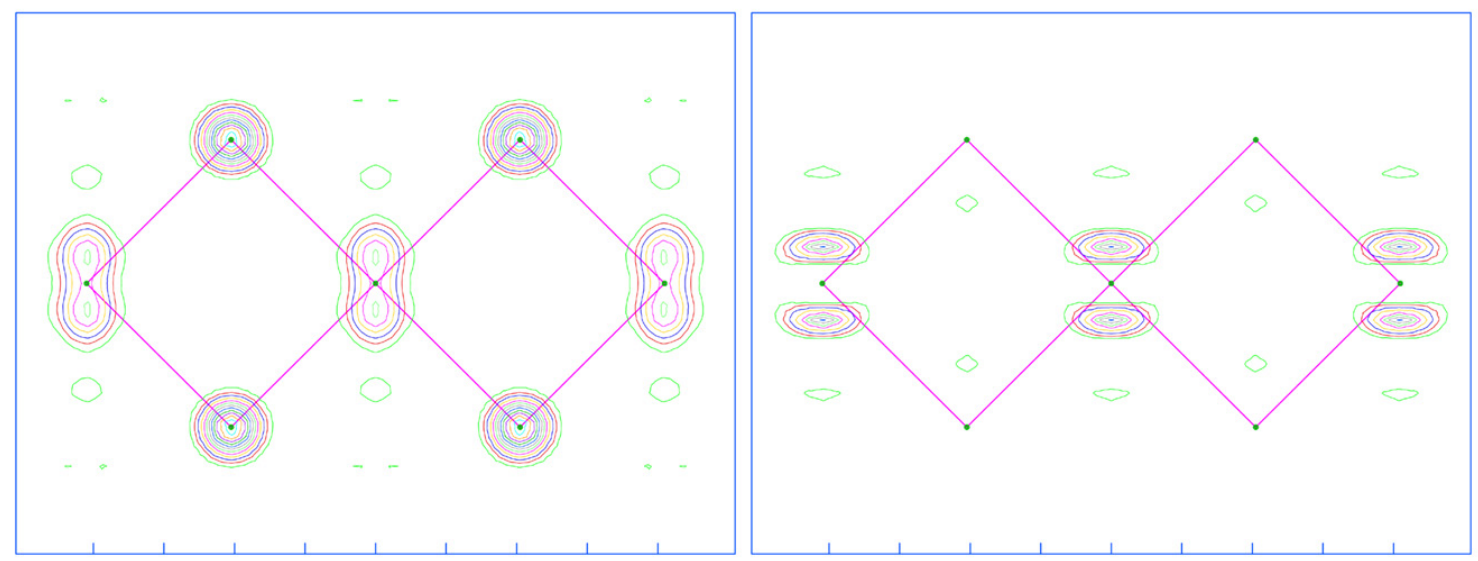

(a)

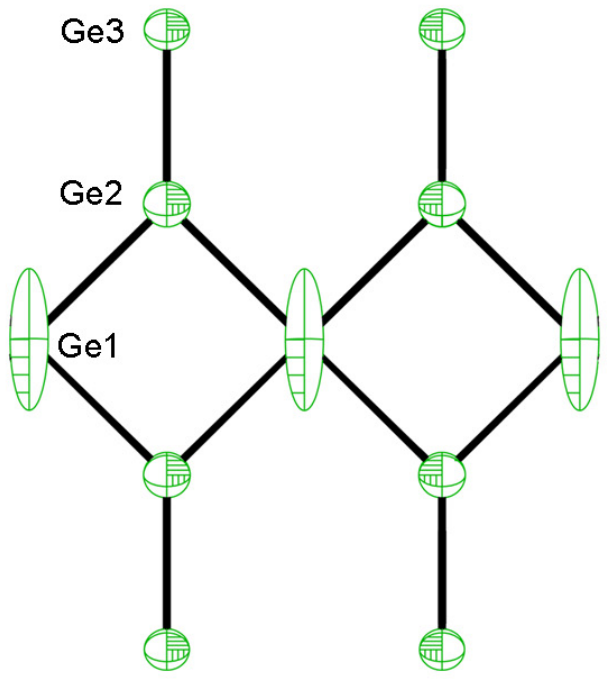

(b)

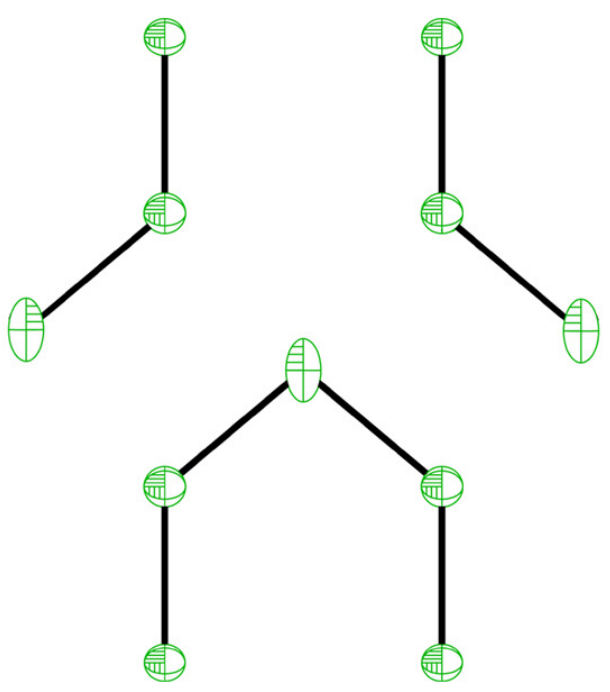

(c)

Figure S3

(a) Plots of the Fourier and the corresponding difference Fourier maps when the $\mathrm{Gd}_{3} \mathrm{Ge}_{4}$ structure is refined isotropically - contour levels at 5 and $2 \mathrm{e}^{-} / \AA^{3}$, respectively. The same fragment of the structure is also drawn with anisotropic displacement parameters (90\% probability). Refinement results using the $\mathrm{Er}_{3} \mathrm{Ge}_{4}$ type as a model $(\mathrm{Ge} 1$ at $0,0,0)$ are shown in (b); the corresponding refinement where Ge1 is offset by about $0.3 \AA$ yields more reasonable distances and thermal parameters (c). See text for details. 


\section{Supporting Information}
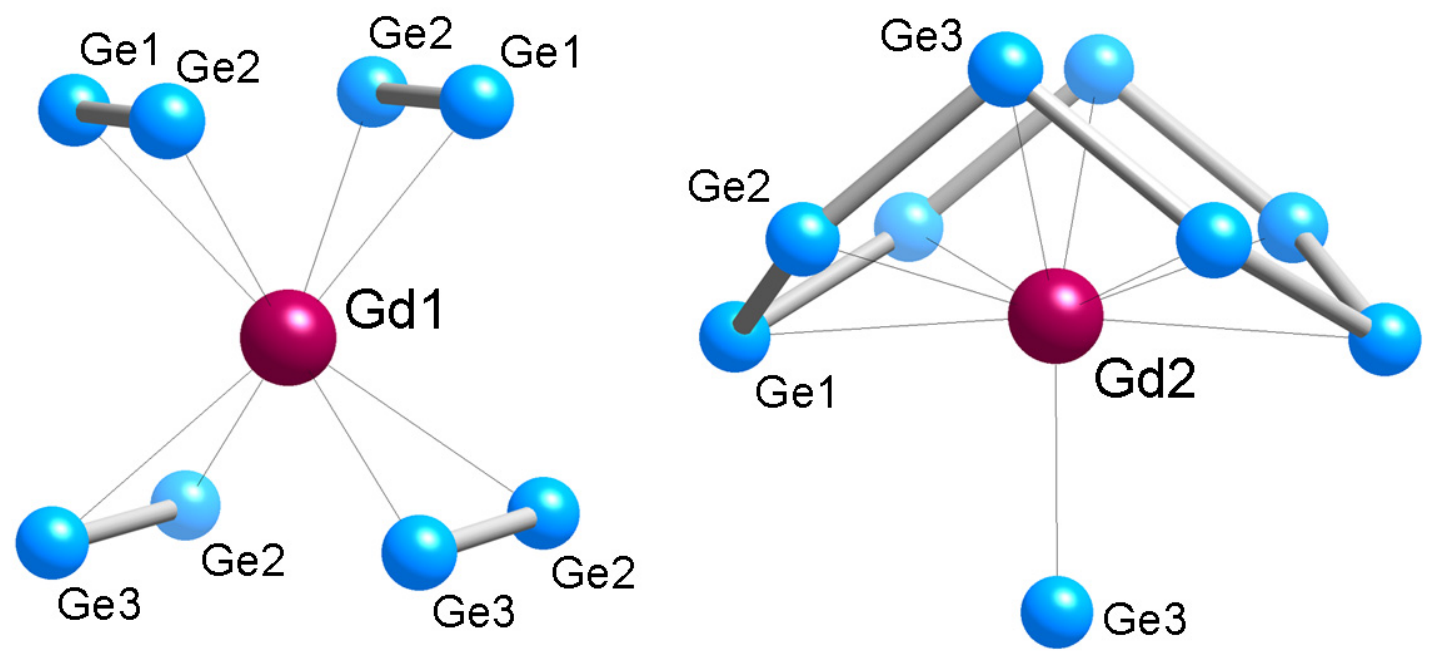

\section{Figure S4}

Coordination polyhedra around the $\mathrm{Gd}$ atoms in the structure of $\mathrm{Gd}_{3} \mathrm{Ge}_{4}$. The $\mathrm{Gd} 1$ cations in the $\mathrm{Gd}_{3} \mathrm{Ge}_{4}$ structure are connected to eight nearest neighbor Ge atoms at distances 2.999(1)-3.107(2) A (Figure 4). The Gd2 cations are surrounded by nine Ge atoms with distances ranging from 2.935(2) to 3.313(2) $\AA$. All Gd-Ge contacts compare well with those reported for other Gd-Ge binary phases such as $\mathrm{Gd}_{5} \mathrm{Ge}_{4},{ }^{3 \mathrm{a}}$ GdGe, ${ }^{17}$ and $\mathrm{Gd}_{3} \mathrm{Ge}_{5}$ for instance. ${ }^{6}$ 


\section{Supporting Information}
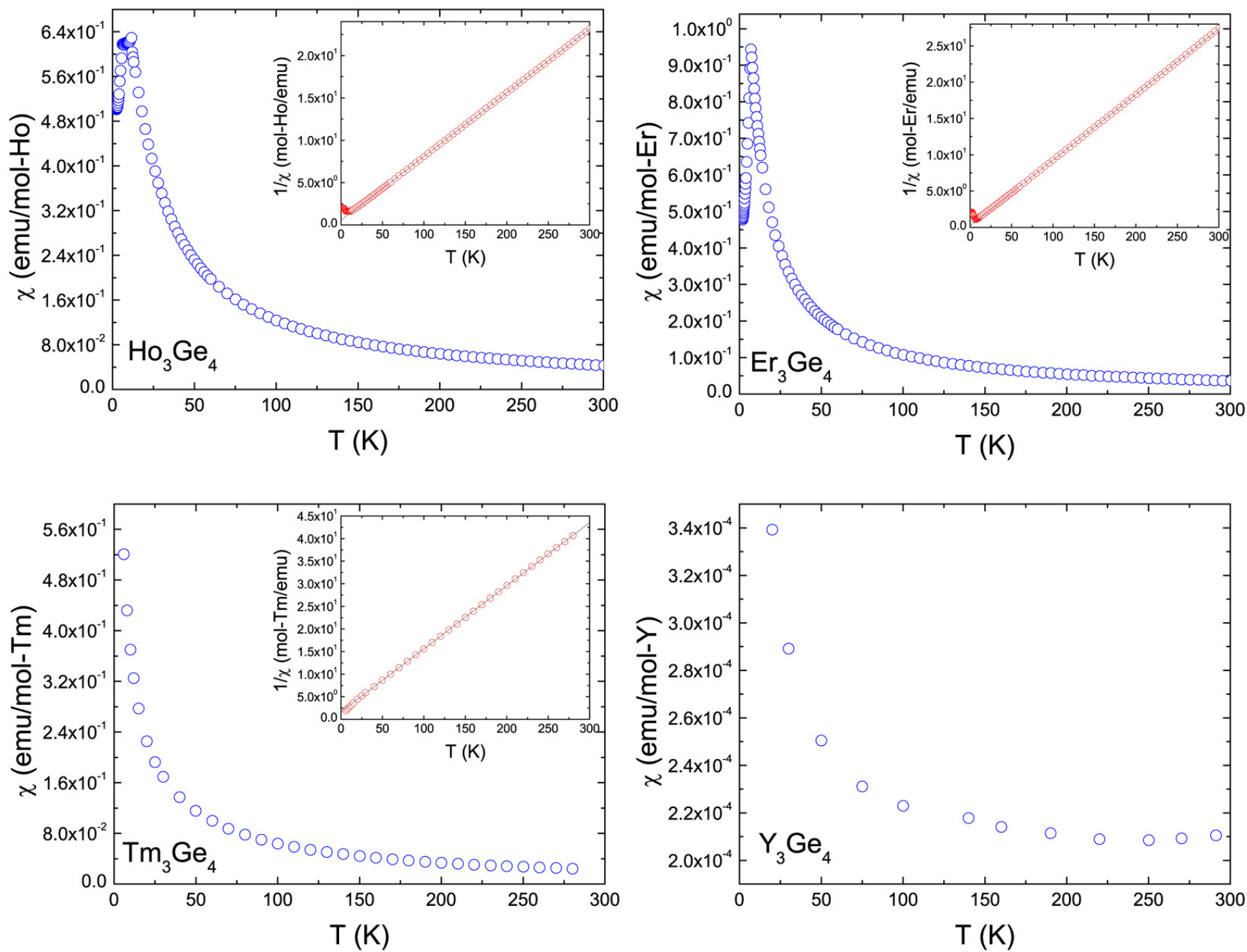

Figure S5

Temperature dependence of the magnetic susceptibility of polycrystalline $\mathrm{RE}_{3} \mathrm{Ge}_{4}(\mathrm{RE}=\mathrm{Y}, \mathrm{Ho}, \mathrm{Er}, \mathrm{Tm})$ measured at an applied field of 500 Oe. The insets show the inverse susceptibility as a function of the temperature. The calculated effective moments are in excellent agreement with the results from previous work. 


\section{Supporting Information}

Table S1. The $h k l$ indices, their $2 \theta$ values and the calculated intensities for $\mathrm{Gd}_{3} \mathrm{Ge}_{4}$.

\begin{tabular}{|c|c|c|c|c|}
\hline $2 \theta(\operatorname{deg})$ & $h$ & $k$ & $l$ & $\mathrm{I}_{\text {calc }}$ \\
\hline 24.05 & 1 & 1 & 1 & 3.6 \\
\hline 24.93 & 0 & 2 & 3 & 18.5 \\
\hline 26.38 & 1 & 1 & 2 & 23.9 \\
\hline 29.87 & 1 & 1 & 3 & 28.8 \\
\hline 33.22 & 1 & 3 & 0 & 82.9 \\
\hline 33.95 & 0 & 4 & 1 & 30.2 \\
\hline 34.25 & 1 & 1 & 4 & 100 \\
\hline 35.56 & 1 & 3 & 2 & 7.2 \\
\hline 37.62 & 0 & 0 & 6 & 25 \\
\hline 38.32 & 1 & 3 & 3 & 32.2 \\
\hline 41.91 & 1 & 3 & 4 & 8.5 \\
\hline 42.02 & 0 & 4 & 4 & 28.9 \\
\hline 44.19 & 2 & 0 & 0 & 35.7 \\
\hline 44.68 & 1 & 1 & 6 & 2.9 \\
\hline 46.19 & 1 & 3 & 5 & 9.9 \\
\hline 51.05 & 1 & 3 & 6 & 17 \\
\hline 51.69 & 1 & 5 & 3 & 10.8 \\
\hline 52.68 & 0 & 6 & 2 & 4.3 \\
\hline 54.31 & 2 & 2 & 4 & 8.2 \\
\hline 56.47 & 0 & 4 & 7 & 11.5 \\
\hline 56.87 & 2 & 4 & 1 & 13.7 \\
\hline 59.39 & 2 & 0 & 6 & 13.1 \\
\hline
\end{tabular}









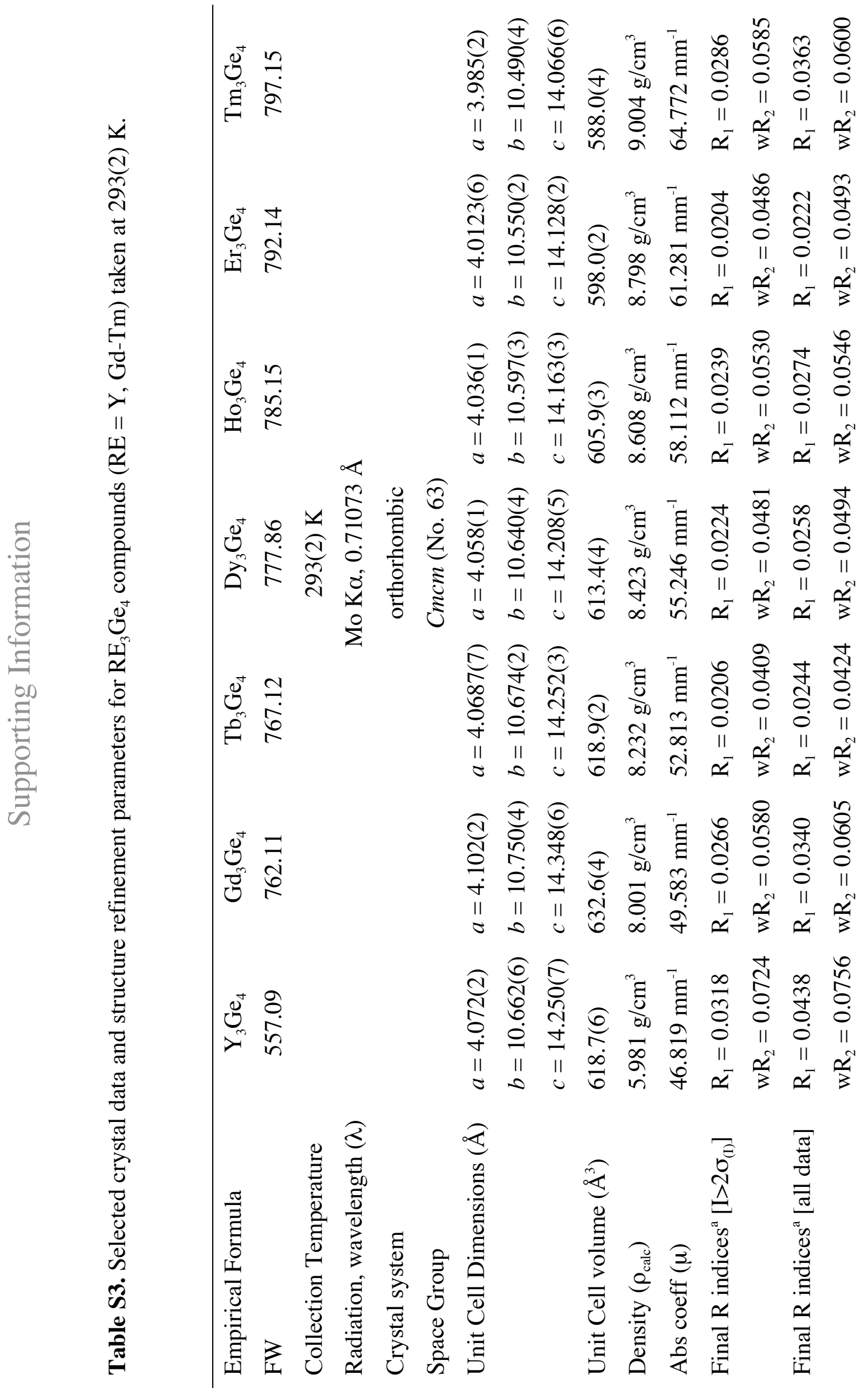




\section{Supporting Information}

Table S4. Selected crystal data and structure refinement parameters for $\mathrm{Gd}_{3} \mathrm{Ge}_{4}$ in $C 2 / c$. The problem with the abnormal thermal parameter for Ge1 persists, although upon lowering the global symmetry from Cmcm to $C 2 / c$, the local site symmetry changes from $2 / m$. . to just an inversion center.

\begin{tabular}{|c|c|}
\hline Empirical formula & $\mathrm{Gd}_{3} \mathrm{Ge}_{4}$ \\
\hline Data collection temperature & 293(2) K \\
\hline Radiation, wavelength $(\lambda)$ & Mo Ka, $0.71073 \AA$ \\
\hline Crystal system & Monoclinic \\
\hline Space group $*$ & $C 2 / c($ No. 15$)$ \\
\hline \multirow[t]{3}{*}{ Unit cell dimensions } & $a=4.102(2) \AA$ \\
\hline & $b=10.750(4) \AA ; \beta=90.03(3)^{\circ}$ \\
\hline & $c=14.348(6) \AA$ \\
\hline Unit cell volume, $\mathrm{Z}$ & $632.7(4) \AA^{3}, 4$ \\
\hline Reflections collected/unique & $1881 / 642\left(\mathrm{R}_{\mathrm{int}}=0.0431\right)$ \\
\hline Parameters & 36 \\
\hline Completeness & $100 \%$ up to $\theta=26.36^{\circ}$ \\
\hline Goodness of fit & 1.04 \\
\hline Final $R$ indices $\left[\mathrm{I}>2 \sigma_{(\mathrm{I})}\right]$ & $\begin{array}{l}\mathrm{R}_{1}=0.0327 \\
\mathrm{wR}_{2}=0.0632\end{array}$ \\
\hline Final $\mathrm{R}$ indices [all data] & $\begin{array}{l}\mathrm{R}_{1}=0.0431 \\
\mathrm{wR}_{2}=0.0671\end{array}$ \\
\hline
\end{tabular}

* The problem with the elongated ADP for one of the Ge atoms is evident in $C 2 / m, C 2$ and even in $P 1$. 


\section{Supporting Information}

Atomic coordinates in $C 2 / c$. we note that the labeling and the atomic coordinates were kept the same so that one can easily track the changes and understand the differences.

\begin{tabular}{llllll}
\hline Atom & $\begin{array}{l}\text { Wyckoff } \\
\text { position }\end{array}$ & $x$ & $y$ & $z$ & $U_{\text {eq }}\left[\AA^{2}\right]$ \\
\hline $\mathrm{Gd} 1$ & $8 f$ & $0.0003(2)$ & $0.3298(1)$ & $0.0968(1)$ & $0.0118(2)$ \\
$\mathrm{Gd} 2$ & $4 e$ & 0 & $0.0428(1)$ & $1 / 4$ & $0.0123(3)$ \\
$\mathrm{Ge} 1^{* *}$ & $4 a$ & 0 & 0 & 0 & $0.0543(10)$ \\
$\mathrm{Ge} 2$ & $8 f$ & $0.0003(4)$ & $0.6185(1)$ & $0.1087(1)$ & $0.0148(3)$ \\
$\mathrm{Ge} 3$ & $4 e$ & 0 & $0.7697(2)$ & $1 / 4$ & $0.0130(4)$ \\
\hline
\end{tabular}

The principal mean square atomic displacements from the refinements in $C 2 / \mathrm{c}$ and in $C m c m$ are compared side-by-side.

\begin{tabular}{|c|c|c|c|c|c|c|c|}
\hline \multicolumn{8}{|c|}{$C 2 / c$} \\
\hline \multicolumn{4}{|c|}{ Ge1 at $0,0,0$} & \multicolumn{4}{|c|}{ Ge1 at $0.002,0.01722,0.01335$} \\
\hline 0.0139 & 0.0117 & 0.0097 & Gd1 & 0.0139 & 0.0116 & 0.0097 & Gd 1 \\
\hline 0.0163 & 0.0109 & 0.0097 & Gd2 & 0.0162 & 0.0109 & 0.0097 & $\mathrm{Gd} 2$ \\
\hline 0.1402 & 0.0123 & 0.0103 & $\mathrm{Ge} 1$ & 0.0506 & 0.0129 & 0.0115 & $\mathrm{Ge} 1$ \\
\hline 0.0163 & 0.0159 & 0.0124 & $\mathrm{Ge} 2$ & 0.0161 & 0.0156 & 0.0126 & $\mathrm{Ge} 2$ \\
\hline 0.0148 & 0.0133 & 0.0110 & Ge3 & 0.0147 & 0.0131 & 0.0112 & $\mathrm{Ge} 3$ \\
\hline \multicolumn{8}{|c|}{$\mathrm{Cmcm}$} \\
\hline \multicolumn{4}{|c|}{ Ge1 at $0,0,0$} & \multicolumn{4}{|c|}{$\mathrm{Ge} 1$ at $0,0.01727,0.01334$} \\
\hline 0.0161 & 0.0106 & 0.0092 & Gd1 & 0.0160 & 0.0107 & 0.0093 & Gd 1 \\
\hline 0.0138 & 0.0114 & 0.0096 & Gd2 & 0.0139 & 0.0113 & 0.0096 & $\mathrm{Gd} 2$ \\
\hline 0.1425 & 0.0116 & 0.0103 & $\mathrm{Ge} 1$ & 0.0519 & 0.0121 & 0.0113 & $\mathrm{Ge} 1$ \\
\hline 0.0144 & 0.0131 & 0.0104 & $\mathrm{Ge} 2$ & 0.0143 & 0.0130 & 0.0105 & $\mathrm{Ge} 2$ \\
\hline 0.0156 & 0.0154 & 0.0122 & $\mathrm{Ge} 3$ & 0.0155 & 0.0152 & 0.0126 & Ge3 \\
\hline
\end{tabular}




\section{Supporting Information}

Table S5. Atomic coordinates and equivalent isotropic displacement parameters for $\mathrm{Y}_{3} \mathrm{Ge}_{4}$ at $120 \mathrm{~K}$.

\begin{tabular}{cccccc}
\hline atom & $\begin{array}{c}\text { Wyckoff } \\
\text { position }\end{array}$ & $x$ & $y$ & $z$ & $U_{\text {eq }}\left(\AA^{2}\right)$ \\
\hline Y1 & $8 f$ & 0 & $0.33027(7)$ & $0.09713(6)$ & $0.0083(3)$ \\
Y2 & $4 c$ & 0 & $0.04543(10)$ & $1 / 4$ & $0.0085(3)$ \\
Ge1 & $\mathbf{8 f}$ & $\mathbf{0}$ & $\mathbf{0 . 0 1 6 8 ( 4 )}$ & $\mathbf{0 . 0 1 3 6 ( 2 )}$ & $\mathbf{0 . 0 1 1 1 ( 8 )}$ \\
Ge2 & $8 f$ & 0 & $0.61975(8)$ & $0.10891(6)$ & $0.0096(3)$ \\
Ge3 & $4 c$ & 0 & $0.77306(10)$ & $1 / 4$ & $0.0083(3)$ \\
& & & Original structure & & \\
Y1 & $8 f$ & 0 & $0.33027(9)$ & $0.09715(8)$ & $0.0087(3)$ \\
Y2 & $4 c$ & 0 & $0.04541(13)$ & $1 / 4$ & $0.0088(4)$ \\
Ge1 & $\mathbf{4 a}$ & $\mathbf{0}$ & $\mathbf{0}$ & $\mathbf{0}$ & $\mathbf{0 . 0 4 1 0}(7)$ \\
Ge2 & $8 f$ & 0 & $0.61974(10)$ & $0.10886(8)$ & $0.0100(3)$ \\
Ge3 & $4 c$ & 0 & $0.77316(13)$ & $1 / 4$ & $0.0085(4)$
\end{tabular}

Table S6. Atomic coordinates and equivalent isotropic displacement parameters for $\mathrm{Tb}_{3} \mathrm{Ge}_{4}$ at $120 \mathrm{~K}$.

\begin{tabular}{cccccc}
\hline & $\begin{array}{c}\text { Wyckoff } \\
\text { position }\end{array}$ & $x$ & $y$ & $z$ & $U_{e q}\left(\AA^{2}\right)$ \\
\hline \multicolumn{5}{c}{ Revised structure } \\
Tb1 & $8 f$ & 0 & $0.33137(4)$ & $0.09691(4)$ & $0.0084(2)$ \\
Tb2 & $4 c$ & 0 & $0.04502(6)$ & $1 / 4$ & $0.0081(2)$ \\
Ge1 & $\mathbf{8 f}$ & $\mathbf{0}$ & $\mathbf{0 . 0 2 0 4}(\mathbf{2})$ & $\mathbf{0 . 0 1 6 4 ( 2 )}$ & $\mathbf{0 . 0 0 8 2}(7)$ \\
$\mathrm{Ge} 2$ & $8 f$ & 0 & $0.62070(10)$ & $0.10911(8)$ & $0.0105(3)$ \\
$\mathrm{Ge} 3$ & $4 c$ & 0 & $0.77248(13)$ & $1 / 4$ & $0.0094(3)$ \\
& & & Original structure & & \\
$\mathrm{Tb} 1$ & $8 f$ & 0 & $0.33143(7)$ & $0.09691(6)$ & $0.0088(4)$ \\
$\mathrm{Tb} 2$ & $4 c$ & 0 & $0.04504(10)$ & $1 / 4$ & $0.0087(4)$ \\
Ge1 & $\mathbf{4 a}$ & $\mathbf{0}$ & $\mathbf{0}$ & $\mathbf{0}$ & $\mathbf{0 . 0 5 9}(2)$ \\
$\mathrm{Ge} 2$ & $8 f$ & 0 & $0.6207(2)$ & $0.10900(14)$ & $0.0110(5)$ \\
$\mathrm{Ge} 3$ & $4 c$ & 0 & $0.7725(2)$ & $1 / 4$ & $0.0098(6)$
\end{tabular}

* $50 \%$ occupied. 


\section{Supporting Information}

Table S7. Atomic coordinates and equivalent isotropic displacement parameters for $\mathrm{Dy}_{3} \mathrm{Ge}_{4}$ at $120 \mathrm{~K}$.

\begin{tabular}{cccccc}
\hline atom & $\begin{array}{c}\text { Wyckoff } \\
\text { position }\end{array}$ & $x$ & $y$ & $z$ & $U_{e q}\left(\AA^{2}\right)$ \\
\hline \multicolumn{5}{c}{ Revised structure } \\
Dy1 & $8 f$ & 0 & $0.33044(6)$ & $0.09712(3)$ & $0.0084(2)$ \\
Dy2 & $4 c$ & 0 & $0.04465(8)$ & $1 / 4$ & $0.0082(3)$ \\
Ge1 $^{*}$ & $\mathbf{8 f}$ & $\mathbf{0}$ & $\mathbf{0 . 0 1 7 0}(\mathbf{6})$ & $\mathbf{0 . 0 1 4 3 ( 4 )}$ & $\mathbf{0 . 0 0 8 9}(\mathbf{1 1})$ \\
Ge2 & $8 f$ & 0 & $0.61992(14)$ & $0.10872(9)$ & $0.0099(4)$ \\
Ge3 & $4 c$ & 0 & $0.77286(2)$ & $1 / 4$ & $0.0091(4)$ \\
& & & Original structure & \\
Dy1 & $8 f$ & 0 & $0.33044(7)$ & $0.09712(5)$ & $0.0087(3)$ \\
Dy2 & $4 c$ & 0 & $0.04471(10)$ & $1 / 4$ & $0.0084(3)$ \\
Ge1 & $\mathbf{4 a}$ & $\mathbf{0}$ & $\mathbf{0}$ & $\mathbf{0}$ & $\mathbf{0 . 0 4 1 0}(\mathbf{1 0})$ \\
Ge2 & $8 f$ & 0 & $0.6200(2)$ & $0.10861(11)$ & $0.0102(4)$ \\
Ge3 & $4 c$ & 0 & $0.7729(2)$ & $1 / 4$ & $0.0092(5)$
\end{tabular}

Table S8. Atomic coordinates and equivalent isotropic displacement parameters for $\mathrm{Ho}_{3} \mathrm{Ge}_{4}$ at $120 \mathrm{~K}$.

\begin{tabular}{cccccc}
\hline & $\begin{array}{c}\text { Wyckoff } \\
\text { position }\end{array}$ & $x$ & $y$ & $z$ & $U_{e q}\left(\AA^{2}\right)$ \\
\hline Ho1 & $8 f$ & 0 & $0.33034(4)$ & $0.09719(3)$ & $0.0083(2)$ \\
Ho2 & $4 c$ & 0 & $0.04521(6)$ & $1 / 4$ & $0.0081(2)$ \\
Ge1 $^{*}$ & $\mathbf{8 f}$ & $\mathbf{0}$ & $\mathbf{0 . 0 1 5 9}(\mathbf{9})$ & $\mathbf{0 . 0 0 9 9}(\mathbf{8})$ & $\mathbf{0 . 0 1 1 8}(\mathbf{1 3})$ \\
Ge2 & $8 f$ & 0 & $0.61985(10)$ & $0.10854(7)$ & $0.0099(3)$ \\
Ge3 & $4 c$ & 0 & $0.77394(14)$ & $1 / 4$ & $0.0090(3)$ \\
& & & Original structure & & \\
Ho1 & $8 f$ & 0 & $0.33034(5)$ & $0.09720(3)$ & $0.0083(2)$ \\
Ho2 & $4 c$ & 0 & $0.04523(6)$ & $1 / 4$ & $0.0081(2)$ \\
Ge1 & $4 a$ & $\mathbf{0}$ & $\mathbf{0}$ & $\mathbf{0}$ & $\mathbf{0 . 0 3 1 2 ( 6 )}$ \\
Ge2 & $8 f$ & 0 & $0.61988(11)$ & $0.10851(8)$ & $0.0099(3)$ \\
Ge3 & $4 c$ & 0 & $0.7739(2)$ & $1 / 4$ & $0.0090(4)$
\end{tabular}

* $50 \%$ occupied. 


\section{Supporting Information}

Table S9. Atomic coordinates and equivalent isotropic displacement parameters for $\mathrm{Er}_{3} \mathrm{Ge}_{4}$ at $120 \mathrm{~K}$.

\begin{tabular}{cccccc}
\hline atom & $\begin{array}{c}\text { Wyckoff } \\
\text { position }\end{array}$ & $x$ & $y$ & $z$ & $U_{e q}\left(\AA^{2}\right)$ \\
\hline Er1 & $8 f$ & 0 & $0.33092(6)$ & $0.09707(5)$ & $0.0084(2)$ \\
Er2 & $4 c$ & 0 & $0.04631(9)$ & $1 / 4$ & $0.0082(3)$ \\
Ge1 & $\mathbf{8 f}$ & $\mathbf{0}$ & $\mathbf{0 . 0 1 5 ( 2 )}$ & $\mathbf{0 . 0 0 9 ( 2 )}$ & $\mathbf{0 . 0 1 0}(2)$ \\
Ge2 & $8 f$ & 0 & $0.6205(2)$ & $0.10863(11)$ & $0.0103(4)$ \\
Ge3 & $4 c$ & 0 & $0.7754(2)$ & $1 / 4$ & $0.0094(5)$ \\
& & & Original structure & & \\
Er1 & $8 f$ & 0 & $0.33092(6)$ & $0.09708(5)$ & $0.0085(3)$ \\
Er2 & $4 c$ & 0 & $0.04634(9)$ & $1 / 4$ & $0.0083(3)$ \\
Ge1 & $\mathbf{4 a}$ & $\mathbf{0}$ & $\mathbf{0}$ & $\mathbf{0}$ & $\mathbf{0 . 0 2 5 5 ( 7 )}$ \\
Ge2 & $8 f$ & 0 & $0.6205(2)$ & $0.10861(11)$ & $0.0104(4)$ \\
Ge3 & $4 c$ & 0 & $0.7754(4)$ & $1 / 4$ & $0.0094(5)$
\end{tabular}

Table S10. Atomic coordinates and equivalent isotropic displacement parameters for $\mathrm{Tm}_{3} \mathrm{Ge}_{4}$ at $120 \mathrm{~K}$.

\begin{tabular}{cccccc}
\hline & $\begin{array}{c}\text { Wyckoff } \\
\text { position }\end{array}$ & $x$ & $y$ & $z$ & $U_{e q}\left(\AA^{2}\right)$ \\
\hline \multicolumn{5}{c}{ atom } & \multicolumn{5}{c}{ Revised structure } \\
Tm1 & $8 f$ & 0 & $0.33156(10)$ & $0.09708(8)$ & $0.0086(4)$ \\
Tm2 & $4 c$ & 0 & $0.04742(13)$ & $1 / 4$ & $0.0080(4)$ \\
Ge1 & $\mathbf{8 f}$ & $\mathbf{0}$ & $\mathbf{0 . 0 1 0}$ & $\mathbf{0 . 0 0 9}$ & $\mathbf{0 . 0 1}(\mathbf{1})$ \\
$\mathrm{Ge} 2$ & $8 f$ & 0 & $0.6212(2)$ & $0.1084(2)$ & $0.0100(6)$ \\
$\mathrm{Ge} 3$ & $4 c$ & 0 & $0.7769(3)$ & $1 / 4$ & $0.0090(8)$ \\
& & & Original structure & & \\
$\mathrm{Tm} 1$ & $8 f$ & 0 & $0.33156(13)$ & $0.09711(10)$ & $0.0072(5)$ \\
$\mathrm{Tm} 2$ & $4 c$ & 0 & $0.0477(2)$ & $1 / 4$ & $0.0071(5)$ \\
Ge1 & $\mathbf{4 a}$ & $\mathbf{0}$ & $\mathbf{0}$ & $\mathbf{0}$ & $\mathbf{0 . 0 1 8 5}(\mathbf{1 2})$ \\
$\mathrm{Ge} 2$ & $8 f$ & 0 & $0.6211(3)$ & $0.1083(2)$ & $0.0082(8)$ \\
$\mathrm{Ge} 3$ & $4 c$ & 0 & $0.7767(4)$ & $1 / 4$ & $0.0076(10)$
\end{tabular}

* $50 \%$ occupied.

** 50\% occupied. The atomic positions were refined when Gel was treated with isotropic displacement parameter, and were then constrained in the anisotropic refinement. 


\section{Supporting Information}

Table S11. Atomic coordinates and equivalent isotropic displacement parameters for $\mathrm{Y}_{3} \mathrm{Ge}_{4}$ at $293 \mathrm{~K}$.

\begin{tabular}{cccccc}
\hline atom & $\begin{array}{c}\text { Wyckoff } \\
\text { position }\end{array}$ & $x$ & $y$ & $z$ & $U_{\text {eq }}\left(\AA^{2}\right)$ \\
\hline Y1 & & \multicolumn{5}{c}{ Revised structure } \\
Y2 & $4 f$ & 0 & $0.32986(10)$ & $0.09721(8)$ & $0.0115(4)$ \\
Ge1 & $4 c$ & 0 & $0.0452(2)$ & $1 / 4$ & $0.0113(4)$ \\
Ge2 & $\mathbf{8 f}$ & $\mathbf{0}$ & $\mathbf{0 . 0 1 5 3 ( 1 2 )}$ & $\mathbf{0 . 0 0 9 4}(\mathbf{1 1})$ & $\mathbf{0 . 0 1 4 ( 2 )}$ \\
$\mathrm{Ge} 3$ & $8 f$ & 0 & $0.61952(12)$ & $0.10877(8)$ & $0.0126(4)$ \\
& $4 c$ & 0 & $0.7730(2)$ & $1 / 4$ & $0.0115(4)$ \\
Y1 & & & Original structure & & \\
Y2 & $4 f$ & 0 & $0.32985(10)$ & $0.09721(8)$ & $0.0116(4)$ \\
Ge1 & $4 a$ & 0 & $0.0452(2)$ & $1 / 4$ & $0.0115(4)$ \\
Ge2 & $8 f$ & $\mathbf{0}$ & $\mathbf{0}$ & $\mathbf{0}$ & $\mathbf{0 . 0 3 1 0}(\mathbf{6})$ \\
$\mathrm{Ge} 3$ & $4 c$ & 0 & $0.61952(12)$ & $0.10875(8)$ & $0.0128(4)$ \\
& & 0 & $0.7730(2)$ & $1 / 4$ & $0.0117(5)$
\end{tabular}

Table S12. Atomic coordinates and equivalent isotropic displacement parameters for $\mathrm{Gd}_{3} \mathrm{Ge}_{4}$ at $293 \mathrm{~K}$.

\begin{tabular}{cccccc}
\hline & $\begin{array}{c}\text { Wyckoff } \\
\text { position }\end{array}$ & $x$ & $y$ & $z$ & $U_{e q}\left(\AA^{2}\right)$ \\
\hline \multicolumn{5}{c}{ Revised structure } \\
Gd1 & $8 f$ & 0 & $0.32975(6)$ & $0.09680(5)$ & $0.0117(3)$ \\
Gd2 & $4 c$ & 0 & $0.04276(8)$ & $1 / 4$ & $0.0122(3)$ \\
Ge1 & $\mathbf{8 f}$ & $\mathbf{0}$ & $\mathbf{0 . 0 1 7 5}(\mathbf{1 0})$ & $\mathbf{0 . 0 1 3 2 ( 8 )}$ & $\mathbf{0 . 0 2 5}(\mathbf{2})$ \\
$\mathrm{Ge} 2$ & $8 f$ & 0 & $0.61853(14)$ & $0.10883(10)$ & $0.0145(4)$ \\
$\mathrm{Ge} 3$ & $4 c$ & 0 & $0.7696(2)$ & $1 / 4$ & $0.0129(5)$ \\
& & & Original structure & & \\
$\mathrm{Gd} 1$ & $8 f$ & 0 & $0.32975(6)$ & $0.09681(5)$ & $0.0117(3)$ \\
$\mathrm{Gd} 2$ & $4 c$ & 0 & $0.04278(9)$ & $1 / 4$ & $0.0122(3)$ \\
Ge1 & $\mathbf{4 a}$ & $\mathbf{0}$ & $\mathbf{0}$ & $\mathbf{0}$ & $\mathbf{0 . 0 5 4 7 ( 1 1 )}$ \\
$\mathrm{Ge} 2$ & $8 f$ & 0 & $0.61854(14)$ & $0.10877(11)$ & $0.0146(4)$ \\
$\mathrm{Ge} 3$ & $4 c$ & 0 & $0.7697(2)$ & $1 / 4$ & $0.0130(5)$
\end{tabular}

* $50 \%$ occupied. 


\section{Supporting Information}

Table S13. Atomic coordinates and equivalent isotropic displacement parameters for $\mathrm{Tb}_{3} \mathrm{Ge}_{4}$ at $293 \mathrm{~K}$.

\begin{tabular}{cccccc}
\hline atom & $\begin{array}{c}\text { Wyckoff } \\
\text { position }\end{array}$ & $x$ & $y$ & $z$ & $U_{e q}\left(\AA^{2}\right)$ \\
\hline \multicolumn{5}{c}{ Revised structure } \\
Tb1 & $8 f$ & 0 & $0.33046(5)$ & $0.09688(3)$ & $0.0115(2)$ \\
$\mathrm{Tb} 2$ & $4 c$ & 0 & $0.04424(7)$ & $1 / 4$ & $0.0115(2)$ \\
Ge1 & $\mathbf{8 f}$ & $\mathbf{0}$ & $\mathbf{0 . 0 1 5 9}(7)$ & $\mathbf{0 . 0 1 1 0}(7)$ & $\mathbf{0 . 0 1 5 8 ( 1 2 )}$ \\
$\mathrm{Ge} 2$ & $8 f$ & 0 & $0.61965(10)$ & $0.10896(8)$ & $0.0135(3)$ \\
$\mathrm{Ge} 3$ & $4 c$ & 0 & $0.7718(2)$ & $1 / 4$ & $0.0122(3)$ \\
& & & Original structure & & \\
$\mathrm{Tb} 1$ & $8 f$ & 0 & $0.33047(5)$ & $0.09688(3)$ & $0.0113(2)$ \\
$\mathrm{Tb} 2$ & $4 c$ & 0 & $0.04426(6)$ & $1 / 4$ & $0.0113(2)$ \\
Ge1 & $\mathbf{4 a}$ & $\mathbf{0}$ & $\mathbf{0}$ & $\mathbf{0}$ & $\mathbf{0 . 0 3 7 8}(\mathbf{6})$ \\
$\mathrm{Ge} 2$ & $8 f$ & 0 & $0.61968(10)$ & $0.10890(8)$ & $0.0132(3)$ \\
$\mathrm{Ge} 3$ & $4 c$ & 0 & $0.77186(14)$ & $1 / 4$ & $0.0120(3)$
\end{tabular}

Table S14. Atomic coordinates and equivalent isotropic displacement parameters for $\mathrm{Dy}_{3} \mathrm{Ge}_{4}$ at $293 \mathrm{~K}$.

\begin{tabular}{cccccc}
\hline atom & $\begin{array}{c}\text { Wyckoff } \\
\text { position }\end{array}$ & $x$ & $y$ & $z$ & $U_{\text {eq }}\left(\AA^{2}\right)$ \\
\hline \multicolumn{5}{c}{ Revised structure } \\
Dy1 & $8 f$ & 0 & $0.32985(5)$ & $0.09711(3)$ & $0.0116(2)$ \\
Dy2 & $4 c$ & 0 & $0.04439(7)$ & $1 / 4$ & $0.0114(2)$ \\
Ge1 & $\mathbf{8 f}$ & $\mathbf{0}$ & $\mathbf{0 . 0 1 3 ( 2 )}$ & $\mathbf{0 . 0 0 9}(\mathbf{2})$ & $\mathbf{0 . 0 1 9}(\mathbf{2})$ \\
Ge2 & $8 f$ & 0 & $0.61924(12)$ & $0.10869(8)$ & $0.0129(3)$ \\
Ge3 & $4 c$ & 0 & $0.7726(2)$ & $1 / 4$ & $0.0117(4)$ \\
& & & Original structure & & \\
Dy1 & $8 f$ & 0 & $0.32985(5)$ & $0.09711(3)$ & $0.0116(2)$ \\
Dy2 & $4 c$ & 0 & $0.04440(7)$ & $1 / 4$ & $0.0114(2)$ \\
Ge1 & $\mathbf{4 a}$ & $\mathbf{0}$ & $\mathbf{0}$ & $\mathbf{0}$ & $\mathbf{0 . 0 3 2 5}(\mathbf{6})$ \\
Ge2 & $8 f$ & 0 & $0.61925(12)$ & $0.10867(8)$ & $0.0129(3)$ \\
Ge3 & $4 c$ & 0 & $0.7726(2)$ & $1 / 4$ & $0.0117(4)$
\end{tabular}

*50\% occupied. 


\section{Supporting Information}

Table S15. Atomic coordinates and equivalent isotropic displacement parameters for $\mathrm{Ho}_{3} \mathrm{Ge}_{4}$ at $293 \mathrm{~K}$.

\begin{tabular}{cccccc}
\hline atom & $\begin{array}{c}\text { Wyckoff } \\
\text { position }\end{array}$ & $x$ & $y$ & $z$ & $U_{\text {eq }}\left(\AA^{2}\right)$ \\
\hline Ho1 & & \multicolumn{5}{c}{ Revised structure } \\
Ho2 & $4 f$ & 0 & $0.33031(5)$ & $0.09705(4)$ & $0.0116(2)$ \\
Ge1 & $4 c$ & 0 & $0.04535(7)$ & $1 / 4$ & $0.0115(3)$ \\
Ge2 & $\mathbf{8 f}$ & $\mathbf{0}$ & $\mathbf{0 . 0 1 4 ( 2 )}$ & $\mathbf{0 . 0 0 9 8 ( 1 4 )}$ & $\mathbf{0 . 0 1 6 ( 2 )}$ \\
Ge3 & $8 f$ & 0 & $0.61972(12)$ & $0.10865(9)$ & $0.0136(3)$ \\
& $4 c$ & 0 & $0.7740(2)$ & $1 / 4$ & $0.0124(4)$ \\
Ho1 & $8 f$ & 0 & $0.33031(5)$ & $0.09705(4)$ & $0.0116(2)$ \\
Ho2 & $4 c$ & 0 & $0.04536(7)$ & $1 / 4$ & $0.0115(3)$ \\
Ge1 & $\mathbf{4 a}$ & $\mathbf{0}$ & $\mathbf{0}$ & $\mathbf{0}$ & $\mathbf{0 . 0 3 1 0 ( 6 )}$ \\
Ge2 & $8 f$ & 0 & $0.61975(12)$ & $0.10863(9)$ & $0.0136(3)$ \\
Ge3 & $4 c$ & 0 & $0.7740(2)$ & $1 / 4$ & $0.0124(4)$
\end{tabular}

Table S16. Atomic coordinates and equivalent isotropic displacement parameters for $\mathrm{Er}_{3} \mathrm{Ge}_{4}$ at $293 \mathrm{~K}$.

\begin{tabular}{cccccc}
\hline atom & $\begin{array}{c}\text { Wyckoff } \\
\text { position }\end{array}$ & $x$ & $y$ & $z$ & $U_{e q}\left(\AA^{2}\right)$ \\
\hline Er1 & $8 f$ & 0 & $0.33121(4)$ & $0.09704(3)$ & $0.0105(2)$ \\
Er2 & $4 c$ & 0 & $0.04676(6)$ & $1 / 4$ & $0.0105(2)$ \\
Ge1 & $\mathbf{8 f}$ & $\mathbf{0}$ & $\mathbf{0 . 0 1 3 2 ( 1 3 )}$ & $\mathbf{0 . 0 0 9 5}(\mathbf{1 0})$ & $\mathbf{0 . 0 1 6}(\mathbf{2})$ \\
$\mathrm{Ge} 2$ & $8 f$ & 0 & $0.62046(10)$ & $0.10865(7)$ & $0.0120(3)$ \\
$\mathrm{Ge} 3$ & $4 c$ & 0 & $0.77608(13)$ & $1 / 4$ & $0.0109(3)$ \\
& & & Original structure & & \\
Er1 & $8 f$ & 0 & $0.33121(4)$ & $0.09704(3)$ & $0.0105(2)$ \\
Er2 & $4 c$ & 0 & $0.04676(6)$ & $1 / 4$ & $0.0104(2)$ \\
Ge1 & $\mathbf{4 a}$ & $\mathbf{0}$ & $\mathbf{0}$ & $\mathbf{0}$ & $\mathbf{0 . 0 3 0 2 ( 5 )}$ \\
Ge2 & $8 f$ & 0 & $0.62049(10)$ & $0.10865(7)$ & $0.0120(3)$ \\
$\mathrm{Ge} 3$ & $4 c$ & 0 & $0.77603(13)$ & $1 / 4$ & $0.0109(3)$
\end{tabular}

*50\% occupied. 


\section{Supporting Information}

Table S17. Atomic coordinates and equivalent isotropic displacement parameters for $\mathrm{Tm}_{3} \mathrm{Ge}_{4}$ at $293 \mathrm{~K}$.

\begin{tabular}{cccccc}
\hline atom & $\begin{array}{c}\text { Wyckoff } \\
\text { position }\end{array}$ & $x$ & $y$ & $z$ & $U_{\text {eq }}\left(\AA^{2}\right)$ \\
\hline Tm1 & $8 f$ & 0 & $0.33159(7)$ & $0.09703(5)$ & $0.0118(3)$ \\
Tm2 & $4 c$ & 0 & $0.04780(10)$ & $1 / 4$ & $0.0116(3)$ \\
Ge1 & $\mathbf{8 f}$ & $\mathbf{0}$ & $\mathbf{0 . 0 1 2 5}$ & $\mathbf{0 . 0 1 1}$ & $\mathbf{0 . 0 1 2 6 ( 8 )}$ \\
$\mathrm{Ge} 2$ & $8 f$ & 0 & $0.6210(2)$ & $0.10843(12)$ & $0.0131(5)$ \\
$\mathrm{Ge} 3$ & $4 c$ & 0 & $0.7775(3)$ & $1 / 4$ & $0.0140(5)$ \\
& & & Original structure & & \\
$\mathrm{Tm} 1$ & $8 f$ & 0 & $0.33160(7)$ & $0.09704(5)$ & $0.0109(3)$ \\
$\mathrm{Tm} 2$ & $4 c$ & 0 & $0.04784(10)$ & $1 / 4$ & $0.0110(3)$ \\
Ge1 & $\mathbf{4 a}$ & $\mathbf{0}$ & $\mathbf{0}$ & $\mathbf{0}$ & $\mathbf{0 . 0 2 7 4 ( 8 )}$ \\
$\mathrm{Ge} 2$ & $8 f$ & 0 & $0.6210(2)$ & $0.10842(12)$ & $0.0133(5)$ \\
$\mathrm{Ge} 3$ & $4 c$ & 0 & $0.7775(3)$ & $1 / 4$ & $0.0122(6)$
\end{tabular}

* 50\% occupied. The atomic positions were refined when Gel was treated with isotropic displacement parameter, and were then constrained in the anisotropic refinement. Despite this difficulty refining the split Gel site, analysis of the Fourier map clearly shows the two maxima:

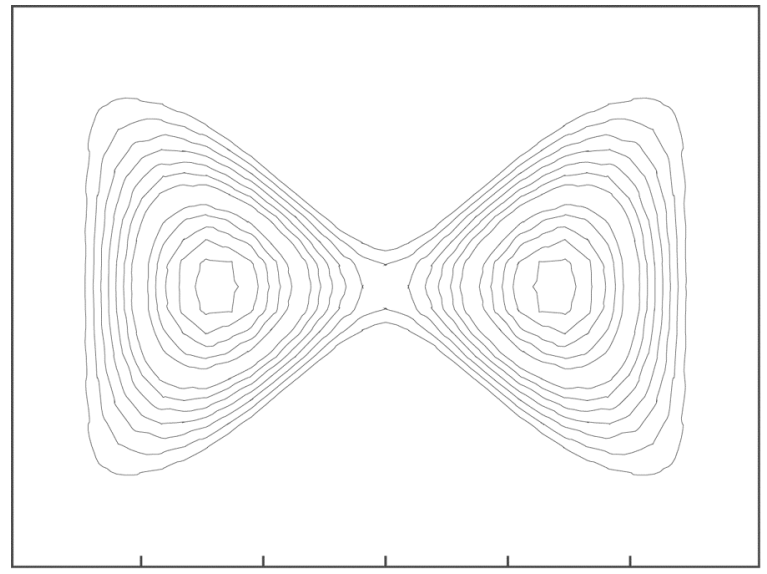

Contour levels at $2 \mathrm{e}^{-} / \AA^{3}$. 


\section{Supporting Information}

Table S18. Selected bond distances in $\mathrm{Y}_{3} \mathrm{Ge}_{4}$ at $120 \mathrm{~K}$.

\begin{tabular}{|c|c|c|c|c|c|}
\hline \multicolumn{2}{|c|}{ atom pair } & distance $(\AA)$ & \multicolumn{2}{|c|}{ atom pair } & distance $(\AA)$ \\
\hline \multicolumn{3}{|c|}{ Revised structure } & \multicolumn{3}{|c|}{ Original structure } \\
\hline \multirow[t]{6}{*}{ Y1- } & Ge2 & $2.974(2)$ & Y1- & $\mathrm{Ge} 2$ & $2.973(2)$ \\
\hline & $\mathrm{Ge} 2 \times 2$ & $3.0247(12)$ & & $\mathrm{Ge} 2 \times 2$ & $3.0247(13)$ \\
\hline & $\mathrm{Ge} 3 \times 2$ & $3.0323(10)$ & & $\mathrm{Ge} 3 \times 2$ & $3.0319(11)$ \\
\hline & $\mathrm{Ge} 1 \times 2$ & $3.0380(5)$ & & Ge1 $1 \times 2$ & $3.0448(10)$ \\
\hline & $\mathrm{Ge} 1 \times 2$ & $3.074(5)$ & & Ge2 & $3.081(2)$ \\
\hline & Ge2 & $3.082(2)$ & Y2- & Ge3 & $2.894(2)$ \\
\hline \multirow[t]{4}{*}{ Y2- } & Ge3 & $2.895(2)$ & & $\mathrm{Ge} 2 \times 4$ & $2.9587(11)$ \\
\hline & $\mathrm{Ge} 2 \times 4$ & $2.9581(10)$ & & $\mathrm{Ge} 3 \times 2$ & $3.158(2)$ \\
\hline & $\mathrm{Ge} 3 \times 2$ & $3.157(2)$ & Ge1- & $\mathrm{Ge} 2 \times 4$ & $2.8500(10)$ \\
\hline & $\mathrm{Ge} 1 \times 2$ & $3.370(5)$ & Ge2- & Ge3 & $2.584(2)$ \\
\hline Ge1- & $\mathrm{Ge} 2 \times 2$ & $2.672(2)$ & & Ge1 $\times 2$ & $2.8500(10)$ \\
\hline \multirow[t]{2}{*}{ Ge2- } & Ge3 & $2.5824(13)$ & Ge3- & $\mathrm{Ge} 2 \times 2$ & $2.584(2)$ \\
\hline & $\mathrm{Ge} 1 \times 2$ & $2.672(2)$ & & & \\
\hline Ge3- & $\mathrm{Ge} 2 \times 2$ & $2.5824(13)$ & & & \\
\hline
\end{tabular}

Table S19. Selected bond distances in $\mathrm{Tb}_{3} \mathrm{Ge}_{4}$ at $120 \mathrm{~K}$.

\begin{tabular}{|c|c|c|c|c|c|}
\hline \multicolumn{2}{|c|}{ atom pair } & distance $(\AA)$ & \multicolumn{2}{|c|}{ atom pair } & distance $(\AA)$ \\
\hline \multicolumn{3}{|c|}{ Revised structure } & \multicolumn{3}{|c|}{ Original structure } \\
\hline \multirow[t]{6}{*}{ Tb1- } & $\mathrm{Ge} 2$ & $2.9790(14)$ & Tb1- & Ge2 & $2.997(2)$ \\
\hline & $\mathrm{Ge} 2 \times 2$ & $3.0339(9)$ & & $\mathrm{Ge} 2 \times 2$ & $3.035(2)$ \\
\hline & $\mathrm{Ge} 1 \times 2$ & $3.0383(2)$ & & Ge $1 \times 2$ & $3.0438(7)$ \\
\hline & $\mathrm{Ge} 3 \times 2$ & $3.0459(6)$ & & $\mathrm{Ge} 3 \times 2$ & $3.0460(9)$ \\
\hline & Ge1 12 & $3.083(2)$ & & $\mathrm{Ge} 2$ & $3.089(2)$ \\
\hline & $\mathrm{Ge} 2$ & $3.0906(13)$ & Tb2- & Ge3 & $2.907(3)$ \\
\hline \multirow[t]{4}{*}{ Tb2- } & Ge3 & $2.907(2)$ & & $\mathrm{Ge} 2 \times 4$ & $2.968(2)$ \\
\hline & $\mathrm{Ge} 2 \times 4$ & $2.9675(9)$ & & $\mathrm{Ge} 3 \times 2$ & $3.164(2)$ \\
\hline & $\mathrm{Ge} 3 \times 2$ & $3.1641(13)$ & Ge1- & $\mathrm{Ge} 2 \times 4$ & $2.8624(14)$ \\
\hline & $\mathrm{Ge} 1 \times 2$ & $3.338(3)$ & Ge2- & Ge3 & $2.580(3)$ \\
\hline Ge1- & $\mathrm{Ge} 2 \times 2$ & $2.648(2)$ & & Ge1 12 & $2.8624(14)$ \\
\hline \multirow[t]{2}{*}{ Ge2- } & Ge3 & $2.578(2)$ & Ge3- & $\mathrm{Ge} 2 \times 2$ & $2.580(3)$ \\
\hline & $\mathrm{Ge} 1 \times 2$ & $2.648(2)$ & & & \\
\hline Ge3- & $\mathrm{Ge} 2 \times 2$ & $2.578(2)$ & & & \\
\hline
\end{tabular}




\section{Supporting Information}

Table S20. Selected bond distances in $\mathrm{Dy}_{3} \mathrm{Ge}_{4}$ at $120 \mathrm{~K}$.

\begin{tabular}{|c|c|c|c|c|c|}
\hline \multicolumn{2}{|c|}{ atom pair } & distance $(\AA)$ & \multicolumn{2}{|c|}{ atom pair } & distance $(\AA)$ \\
\hline \multicolumn{3}{|c|}{ Revised structure } & \multicolumn{3}{|c|}{ Original structure } \\
\hline \multirow[t]{6}{*}{ Dy1- } & $\mathrm{Ge} 2$ & $2.962(2)$ & Dy1- & $\mathrm{Ge} 2$ & $2.960(2)$ \\
\hline & $\mathrm{Ge} 2 \times 2$ & $3.0152(13)$ & & $\mathrm{Ge} 2 \times 2$ & $3.015(2)$ \\
\hline & $\mathrm{Ge} 3 \times 2$ & $3.0231(8)$ & & $\mathrm{Ge} 3 \times 2$ & $3.0231(9)$ \\
\hline & Ge1 $\times 2$ & $3.031(6)$ & & $\mathrm{Ge} 1 \times 2$ & $3.0339(7)$ \\
\hline & $\mathrm{Ge} 1 \times 2$ & $3.061(6)$ & & $\mathrm{Ge} 2$ & $3.075(2)$ \\
\hline & $\mathrm{Ge} 2$ & $3.074(2)$ & Dy2- & Ge3 & $2.882(3)$ \\
\hline \multirow[t]{4}{*}{ Dy2- } & Ge3 & $2.882(2)$ & & $\mathrm{Ge} 2 \times 4$ & $2.9539(13)$ \\
\hline & $\mathrm{Ge} 2 \times 4$ & $2.9528(12)$ & & $\mathrm{Ge} 3 \times 2$ & $3.152(2)$ \\
\hline & $\mathrm{Ge} 3 \times 2$ & $3.152(2)$ & Ge1- & $\mathrm{Ge} 2 \times 4$ & $2.8399(12)$ \\
\hline & Ge1 $\times 2$ & $3.350(5)$ & Ge2- & Ge3 & $2.576(2)$ \\
\hline Ge1- & $\mathrm{Ge} 2 \times 2$ & $2.657(2)$ & & Ge1 $\times 2$ & $2.8399(12)$ \\
\hline \multirow[t]{2}{*}{ Ge2- } & Ge3 & $2.575(2)$ & Ge3- & $\mathrm{Ge} 2 \times 2$ & $2.576(2)$ \\
\hline & $\mathrm{Ge} 1 \times 2$ & $2.657(2)$ & & & \\
\hline Ge3- & $\mathrm{Ge} 2 \times 2$ & $2.575(2)$ & & & \\
\hline
\end{tabular}

Table S21. Selected bond distances in $\mathrm{Ho}_{3} \mathrm{Ge}_{4}$ at $120 \mathrm{~K}$.

\begin{tabular}{|c|c|c|c|c|c|}
\hline \multicolumn{2}{|c|}{ atom pair } & distance $(\AA)$ & \multicolumn{2}{|c|}{ atom pair } & distance $(\AA)$ \\
\hline \multicolumn{3}{|c|}{ Revised structure } & \multicolumn{3}{|c|}{ Original structure } \\
\hline \multirow[t]{6}{*}{ Ho1- } & $\mathrm{Ge} 2$ & $2.956(2)$ & Ho1- & $\mathrm{Ge} 2$ & $2.956(2)$ \\
\hline & Ge1 $1 \times 2$ & $3.000(10)$ & & $\mathrm{Ge} 2 \times 2$ & $3.0078(13)$ \\
\hline & $\mathrm{Ge} 2 \times 2$ & $3.0080(13)$ & & $\mathrm{Ge} 3 \times 2$ & $3.0141(10)$ \\
\hline & $\mathrm{Ge} 3 \times 2$ & $3.0141(10)$ & & Ge1 $\times 2$ & $3.0287(8)$ \\
\hline & Ge2 & $3.068(2)$ & & Ge2 & $3.068(2)$ \\
\hline & $\mathrm{Ge} 1 \times 2$ & $3.073(10)$ & Ho2- & Ge3 & $2.871(2)$ \\
\hline \multirow[t]{4}{*}{ Ho2- } & Ge3 & $2.871(2)$ & & $\mathrm{Ge} 2 \times 4$ & $2.9473(12)$ \\
\hline & $\mathrm{Ge} 2 \times 4$ & $2.9471(11)$ & & $\mathrm{Ge} 3 \times 2$ & $3.150(2)$ \\
\hline & $\mathrm{Ge} 3 \times 2$ & $3.150(2)$ & Ge1- & $\mathrm{Ge} 2 \times 4$ & $2.8327(11)$ \\
\hline & $\mathrm{Ge} 1 \times 2$ & $3.408(11)$ & Ge2- & Ge3 & $2.581(2)$ \\
\hline Ge1- & $\mathrm{Ge} 2 \times 2$ & $2.686(5)$ & & Ge $1 \times 2$ & $2.8327(11)$ \\
\hline \multirow[t]{2}{*}{ Ge2- } & Ge3 & $2.580(2)$ & Ge3- & $\mathrm{Ge} 2 \times 2$ & $2.581(2)$ \\
\hline & $\mathrm{Ge} 1 \times 2$ & $2.686(5)$ & & & \\
\hline Ge3- & $\mathrm{Ge} 2 \times 2$ & $2.580(2)$ & & & \\
\hline
\end{tabular}




\section{Supporting Information}

Table S22. Selected bond distances in $\mathrm{Er}_{3} \mathrm{Ge}_{4}$ at $120 \mathrm{~K}$.

\begin{tabular}{|c|c|c|c|c|c|}
\hline \multicolumn{2}{|c|}{ atom pair } & distance $(\AA)$ & \multicolumn{2}{|c|}{ atom pair } & distance $(\AA)$ \\
\hline \multicolumn{3}{|c|}{ Revised structure } & \multicolumn{3}{|c|}{ Original structure } \\
\hline \multirow[t]{6}{*}{ Er1- } & $\mathrm{Ge} 2$ & $2.940(2)$ & Er1- & $\mathrm{Ge} 2$ & $2.940(2)$ \\
\hline & $\mathrm{Ge} 1 \times 2$ & $2.980(2)$ & & $\mathrm{Ge} 2 \times 2$ & $2.988(2)$ \\
\hline & $\mathrm{Ge} 2 \times 2$ & $2.988(2)$ & & $\mathrm{Ge} 3 \times 2$ & $2.9959(10)$ \\
\hline & $\mathrm{Ge} 3 \times 2$ & $2.9960(10)$ & & $\mathrm{Ge} 1 \times 2$ & $3.0055(8)$ \\
\hline & $\mathrm{Ge} 1 \times 2$ & $3.044(2)$ & & $\mathrm{Ge} 2$ & $3.051(2)$ \\
\hline & $\mathrm{Ge} 2$ & $3.051(2)$ & Er2- & Ge3 & $2.850(3)$ \\
\hline \multirow[t]{4}{*}{ Er2- } & Ge3 & $2.850(3)$ & & $\mathrm{Ge} 2 \times 4$ & $2.9277(14)$ \\
\hline & $\mathrm{Ge} 2 \times 4$ & $2.9276(13)$ & & $\mathrm{Ge} 3 \times 2$ & $3.132(2)$ \\
\hline & $\mathrm{Ge} 3 \times 2$ & $3.132(2)$ & Ge1- & $\mathrm{Ge} 2 \times 4$ & $2.8191(13)$ \\
\hline & Ge1 $\times 2$ & $3.40(2)$ & Ge2- & Ge3 & $2.572(2)$ \\
\hline Ge1- & $\mathrm{Ge} 2 \times 2$ & $2.684(8)$ & & Ge1 $\times 2$ & $2.8191(13)$ \\
\hline \multirow[t]{2}{*}{ Ge2- } & Ge3 & $2.572(2)$ & Ge3- & $\mathrm{Ge} 2 \times 2$ & $2.572(2)$ \\
\hline & $\mathrm{Ge} 1 \times 2$ & $2.684(8)$ & & & \\
\hline Ge3- & $\mathrm{Ge} 2 \times 2$ & $2.572(2)$ & & & \\
\hline
\end{tabular}

Table S23. Selected bond distances in $\mathrm{Tm}_{3} \mathrm{Ge}_{4}$ at $120 \mathrm{~K}$.

\begin{tabular}{|c|c|c|c|c|c|}
\hline \multicolumn{2}{|c|}{ atom pair } & distance $(\AA)$ & \multicolumn{2}{|c|}{ atom pair } & distance $(\AA)$ \\
\hline \multicolumn{3}{|c|}{ Revised structure } & \multicolumn{3}{|c|}{ Original structure } \\
\hline \multirow[t]{6}{*}{ Tm1- } & $\mathrm{Ge} 2$ & $2.927(3)$ & Tm1- & $\mathrm{Ge} 2$ & $2.925(4)$ \\
\hline & $\mathrm{Ge} 2 \times 2$ & $2.976(2)$ & & $\mathrm{Ge} 2 \times 2$ & $2.975(3)$ \\
\hline & $\mathrm{Ge} 3 \times 2$ & $2.982(2)$ & & $\mathrm{Ge} 3 \times 2$ & $2.983(2)$ \\
\hline & $\mathrm{Ge} 1 \times 2$ & $2.998(1)$ & & Ge1 $\times 2$ & $2.9891(13)$ \\
\hline & $\mathrm{Ge} 1 \times 2$ & 2.991(1) & & $\mathrm{Ge} 2$ & $3.039(4)$ \\
\hline & $\mathrm{Ge} 2$ & $3.038(3)$ & $\operatorname{Tm} 2-$ & Ge3 & $2.840(5)$ \\
\hline \multirow[t]{3}{*}{$\operatorname{Tm} 2-$} & Ge3 & $2.835(4)$ & & $\mathrm{Ge} 2 \times 4$ & $2.917(2)$ \\
\hline & $\mathrm{Ge} 2 \times 4$ & $2.916(2)$ & & $\mathrm{Ge} 3 \times 2$ & $3.118(4)$ \\
\hline & $\mathrm{Ge} 3 \times 2$ & $3.122(3)$ & Ge1- & $\mathrm{Ge} 2 \times 4$ & $2.807(2)$ \\
\hline Ge1- & $\mathrm{Ge} 2 \times 2$ & $2.686(2)$ & Ge2- & Ge3 & $2.572(4)$ \\
\hline \multirow[t]{2}{*}{ Ge2- } & Ge3 & $2.572(3)$ & & Ge $1 \times 2$ & $2.807(2)$ \\
\hline & $\mathrm{Ge} 1 \times 2$ & $2.686(2)$ & Ge3- & $\mathrm{Ge} 2 \times 2$ & $2.572(4)$ \\
\hline Ge3- & $\mathrm{Ge} 2 \times 2$ & $2.572(3)$ & & & \\
\hline
\end{tabular}

